\title{
Metals Property Changes Under Effect of Vacancy-Cluster Structures
}

\author{
Viktor Novikov, Mark Levin, Vyacheslav Pevgov, Viktor Ulyanov \\ Department of Innovation Energy Systems “INESYS”, National University of Science and Technology «MISIS», Moscow, Russia
}

Email address:

novikov-nanotech@yandex.ru (V. Novikov), ulyanovik@gmail.com (V. Ulyanov)

\section{To cite this article:}

Viktor Novikov, Mark Levin, Vyacheslav Pevgov, Viktor Ulyanov. Metals Property Changes Under Effect of Vacancy-Cluster Structures. International Journal of Materials Science and Applications. Vol. 8, No. 1, 2019, pp. 1-11. doi: 10.11648/j.ijmsa.20190801.11

Received: April 11, 2019; Accepted: May 30, 2019; Published: June 12, 2019

\begin{abstract}
The experimental results analysis of a metals property changes under vacancy-cluster structure effects are shown. Two technological approaches of such structures obtaining are considered. The first is a nanopowders compaction under high (up to $5 \mathrm{GPa}$ ) hydrostatic compression, on example of a $\mathrm{Ni}$ nanopowder $(70 \mathrm{~nm})$. The second is the $\mathrm{Al}$ and $\mathrm{Pb}$ crystallization under the high-intensity plastic deformation $\left[\varepsilon^{\prime}=\left(10^{2}-10^{4}\right) \mathrm{sec}^{-1}\right]$ (HIPD) conditions on the "solid-liquid" boundary in the centrifugal casting machine with rotary speed up to $2000 \mathrm{rpm}$. Using the method of atomic force microscopy (AFM), vacancy cluster tubes (VCT) with average diameters of $39 \mathrm{~nm}$ for $\mathrm{Al}$ and $25 \mathrm{~nm}$ for $\mathrm{Pb}$ have been detected in the crystallized volume of $\mathrm{Al}$ and $\mathrm{Pb}$ metals. Discussed the physical model of a new substructure formation within the metals in the form of vacancy cluster tubes, obtained in the process of high-intensive plastic deformation (HIPD) during the process of mass crystallization of $\mathrm{Al}$ and $\mathrm{Pb}$ and the changes in the mechanical, magnetic and superconducting properties of the above metals, which followed this process. During $\mathrm{Al}$ and $\mathrm{Pb}$ crystallization under high-intensive plastic deformation (HIPD) range about $\left[\varepsilon^{\prime}=\left(10^{2}-10^{4}\right) \mathrm{sec}^{-}\right.$ ${ }^{1}$ ] with specially selected modes of metals crystallization in high-speed centrifugal casting machine the special conditions are being created to achieve the dimensional effect of dynamic (shifting) re-crystallization. Shifting deformation during centrifugal crystallization caused primarily by a large incline of the temperature field from the periphery (relative to the cold wall of the rotor) to the molten central part of the rotor. The difference in the angular velocities of the already-frozen part of the metal (adjacent to the outer surface of the rotor wall) and the central part, where the metal still remains in the molten state, leads to a high-intensity deformation $\left[\varepsilon^{\prime}=\left(10^{2}-10^{4}\right) \mathrm{sec}^{-1}\right]$ of the crystallized metal melt solidified phase. Since the grain sizes at the crystallized phase initially comprise around tens of nano-meters (approximately crystal nucleation size), it becomes possible to achieve the dimensional effect of the dynamic re-crystallization of a «nanocrystalline» solidified metal at high shift of strain velocities. The «non-equilibrium vacancies》 formed this way condense into vacancy clusters, which are formed in the centrifugal force field in the form of vacancy-shaped cluster tubes stretched out to the center of rotation of the rotor. The process proceeds under conditions far from the equilibrium in comparison with the usual crystallization of the metal from the melt. Such processes can lead to the formation of highly ordered non-equilibrium statescharacteristic of non-equilibrium open systems. Discussed the physical model of a metals vacancy-cluster structures formation at high hydrostatic nanopowders compression (up to $5 \mathrm{GPa}$ ) and high-intensity plastic deformation (HIPD) at the stage of Al and Pb alloys mass crystallization during centrifugation. Conclusion of the article is that the high-intensity plastic deformation (HIPD) at the melt crystallization stage against a background of high stationary nonequilibrium vacancies concentration brings to the new type of the elements structure formation - vacancy cluster tubes (VCT). A comparative analysis of mechanical, magnetic and superconducting properties changes for structured metals introduced.
\end{abstract}

Keywords: Vacancy Clusters, Vacancy-Cluster Structure, Intense Plastic Deformation, Dynamic Recrystallization, Nonequilibrium Vacancies, Vacancy Cluster Tubes, Solid-Phase Recrystallization 


\section{The Vacancy-Clusters Formation in Nano-Disperse Metal Media (NMM) and Their Properties}

The recent years studding's has established a new mechanism for the vacancy-cluster structures formation in nanodispersed metal media (NMM) under severe plastic deformation. This mechanism called as dimensional effect of nanopowders self-activated dynamic recrystallization [1-6]. The description of this effect based on the physical explanations given by S. S. Gorelik [7] and G. Gleiter [8] a few decades ago. They suggested that the excessive (nonequilibrium) vacancies $[9,10]$ occurred during the borders migration. This formation of excess vacancies in the rear of the movement does not represent the usual process of diffusion redistribution of grain boundary emptiness in the grain volume. Apparently, it is better to say a "generation" of nonequilibrium vacancies (creating additional atomic lattice) of kinetic origin. In this case, the birth of superfluous vacancies must accompanied by the effect of positive volume dilation associated with the formation of additional atomic nodes in the recrystallized volume lattice. This experimentally confirmed by studies [11]. In the articles [10, 12] are assumed that the atermic generation of vacancies as a result of the boundaries movements are occurs from the crawling stages of GBD similar to mechanism of the screw dislocation sliding with stairs [12]. The borders migration rate then will be determined by the rate of a point defects (vacancies) "generation" (removal) from the crawling stairs of the GBD. The vacancy "generation" effect for systems with grain (block) sizes smaller than the average diffusion path of dynamic vacancies may have a specific character of the self-activation collective size recrystallization effect [1, 4]. As it is shown $[1,4]$ within a short period of time (about several minutes) the concentration level of dynamic vacancies in NMM can be comparable with the concentration level of thermodynamic vacancies at temperatures close to melting. This correlated with the experiment results [2-6]. The physical meaning of the size effects for self-activated recrystallization is that the dynamic vacancies have time to reach nearby borders and trigger the activation of their movement starting with some critical grain size (Figure 1, $\left.d_{m}\right)[1,4]$. This process characterized by a dimensional effect. In the article [2] on the example of the $\mathrm{Ni} \mathrm{NMM}$ experimentally shown that in the conditions of uniaxial pressing there is a short-term "generation" of superfluous vacancies corresponding to pre-melting temperatures $\left(\sim 10^{-4}\right)$. The possibility of solid-phase recrystallization in the "swept" boundary volume during grain boundary slippage (GBS) of particles under plastic shear conditions leads to the formation of single-crystal "embryos" in the border regions (GBS region) [4].

In this case, the usual notion of a critical embryo is not applicable. Apparently, it is more correctly to say about the "germ-free" mechanism of new grains formation when a significant supersaturation of nonequilibrium vacancies leads to solid-phase recrystallization [2-6]. At the same time, in the zone of high concentration of redundant vacancies, vacancy cluster formations arise. Their faceting corresponded to the metal lattice type (Figure 2) [13].

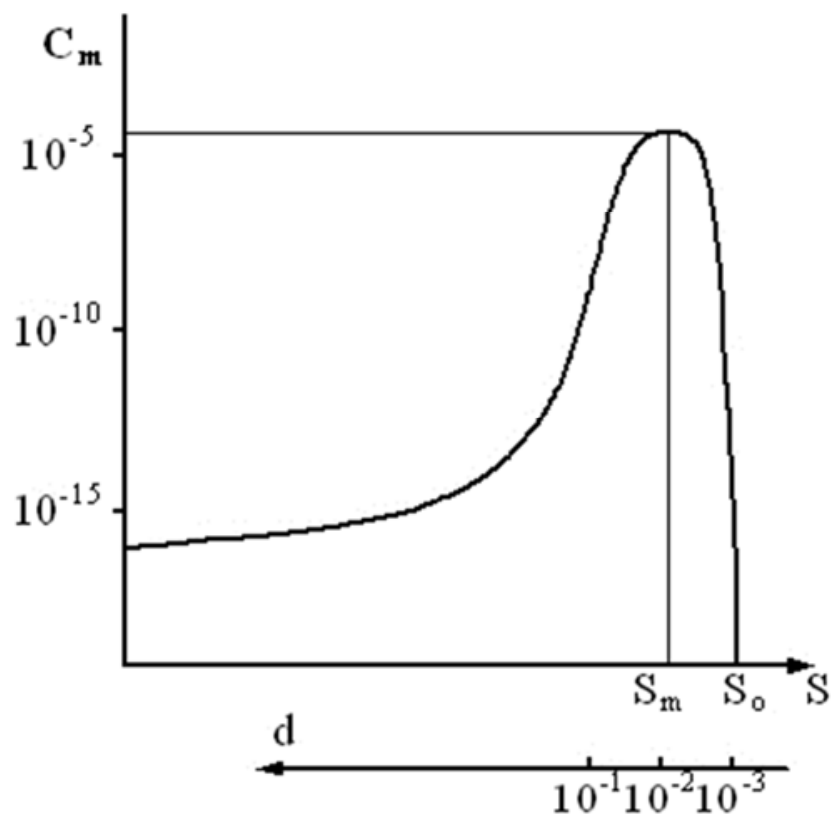

Figure 1. The dependences of the nonequilibrium vacancies concentration $C m$ on grain size $d$ in the boundary migration process $(S=a / d)$.

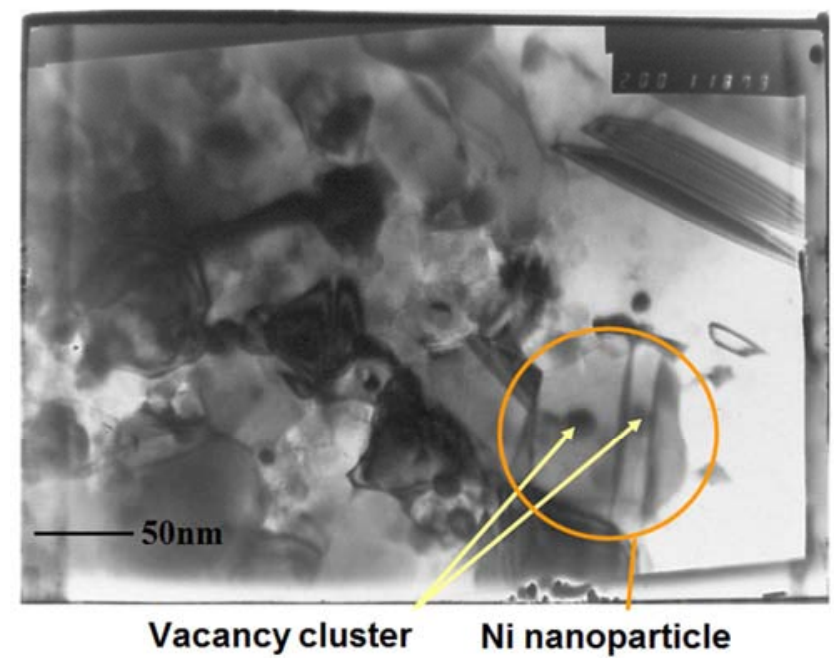

Figure 2. Transmission electron microscopy from a thin slice of $\mathrm{Ni}$ nanocrystalline compact sample which made by high hydrostatic compression of a Ni nanopowder (70nm) at a pressure $5 \mathrm{GPa}$ and a temperature $900^{\circ} \mathrm{C}[13]$.

The effect of vacancy-cluster structures on the metals mechanical properties is considered in these studies [14-16] where studied the stress-strain $(\sigma \sim \varepsilon)$ dependence in the uniaxial compression mode for the Ni nanocrystalline $(70 \mathrm{~nm})$ samples with vacancy clusters formed in the process its nanopowders compaction [13]. The Figure 3 shows the Ni stress-strain curves with a grain size of $100 \mu \mathrm{m}$ (Figure 3a) and nanocrystalline Ni $(70 \mathrm{~nm})$ with vacancy clusters (Figure 3b). 
On the samples of nanocrystalline $\mathrm{Ni}(70 \mathrm{~nm})$ the effect of abnormally high reversible deformation of «pseudoelasticity» after unloading of the sample with value reaches $8 \%$, is observed. The coarse-dispersed Ni $(100 \mu \mathrm{m})$ sample shows the traditional stress-strain relationship $(\sigma \sim \varepsilon)$ for metals. A specific feature of the obtained results in stressstrain curves $(\sigma \sim \varepsilon)$ for $\mathrm{Ni}(70 \mathrm{~nm})$ is the lack of the reloading hardening effect, which is always present in the case of metals with micron grain sizes (Figure3a).
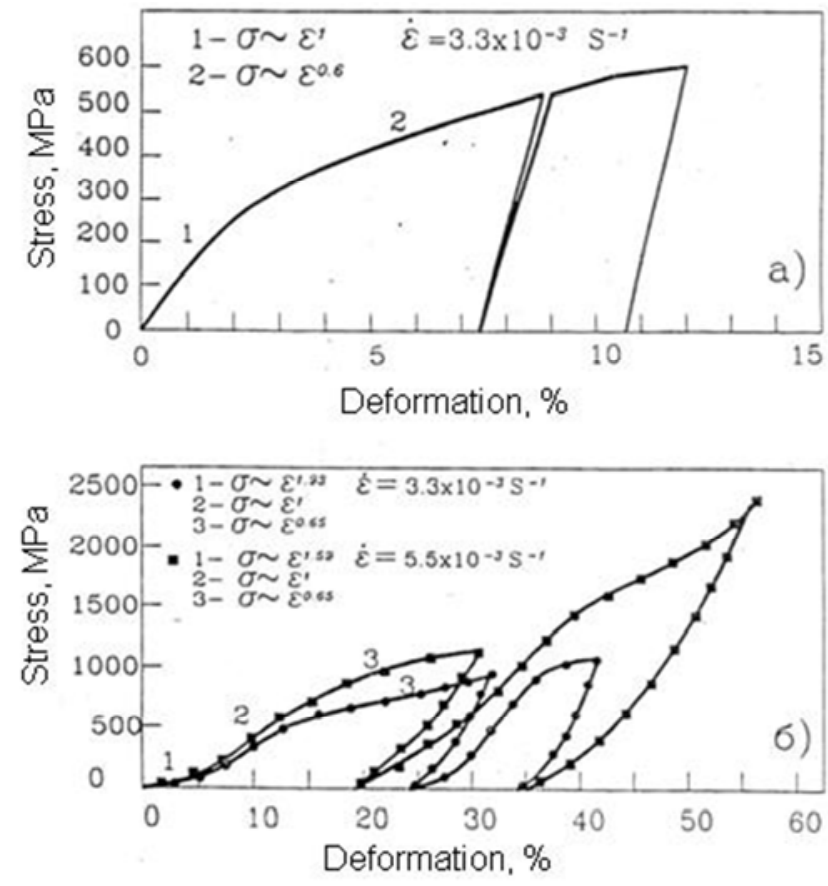

Figure 3. Stress-strain inter-dependence $(\sigma \sim \varepsilon)$ for poly-crystalline nickel with: (a) grain size of $100 \mu \mathrm{m}$, and (b) nano-crystalline nickel $\mathrm{Ni}(70 \mathrm{~nm})$.

The Figure 4 shows stress-strain $(\sigma \sim \varepsilon)$ curves obtained for nanocrystalline $\mathrm{Ni}(70 \mathrm{~nm})$ under uniaxial compression mode at temperatures from $350^{\circ} \mathrm{C}$ to $500^{\circ} \mathrm{C}$ in vacuum $10^{-2}$ $\mathrm{mm} \mathrm{Hg}$. The qualitative character of $(\sigma \sim \varepsilon)$ dependence in the investigated temperature range is maintained. The quantitative changes appearing in reduction of the stress level at the temperature increases. Small hysteresis loop also appears under repeated loading, which is associated with the enlargement of $\mathrm{Ni}$ grains following the growth of temperature (re-crystallization process) to the micron size values. The effect of abnormally high reversible deformation of $\mathrm{Ni}$ with vacancy-cluster structure is preserved. This effect of "pseudoelasticity" is appear in the whole range of investigated temperatures $\left(20^{\circ} \mathrm{C}-500^{\circ} \mathrm{C}\right)$ and reaches $8 \%$ of the linear size of the sample with a total deformation at $55 \%$. Such high strain values of the sample, without its destruction indicates the high plasticity resource of the nano-crystalline Ni $(70 \mathrm{~nm})$. For the Ni (70nm) samples is proposed that the source of the internal elastic stresses, with relaxation of which the anomalously high reversible deformation effect is concerned, serves the field of elastic stresses caused by the interactions of these vacancy clusters $[13,14]$.
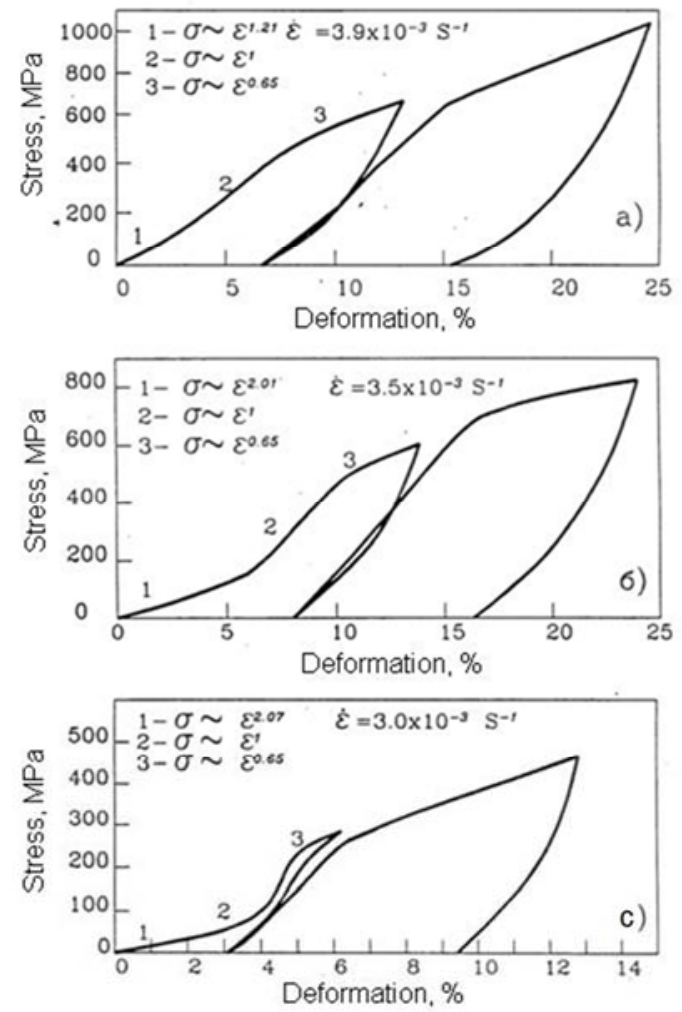

Figure 4. Stress-strain inter-dependence $(\sigma \sim \varepsilon)$ for nano-crystalline nickel $\mathrm{Ni}(70 \mathrm{~nm})$ at different temperatures: a) $-350^{\circ} \mathrm{C}$; b) $-400^{\circ} \mathrm{C}$; c) $-500^{\circ} \mathrm{C}$.

The absence of the hardening effect under repeated loading of nanocrystalline $\mathrm{Ni}(70 \mathrm{~nm})$ samples indicated that innergrain plastic deformation occurs by a mechanism of diffusion-viscous flow without the formation of dislocation structure that changes the form and shape of the curve $(\sigma \sim \varepsilon)$ at the initial stage of deformation [14-16]. As shown by special studies in stronglydeformed NMM the dislocation structure there is not exists [11, 13] (Figure 2) and that is correlated with the theoretical estimates of the dislocation loop $(0.5-1.5 \mu \mathrm{m})$ critical sizes [12].

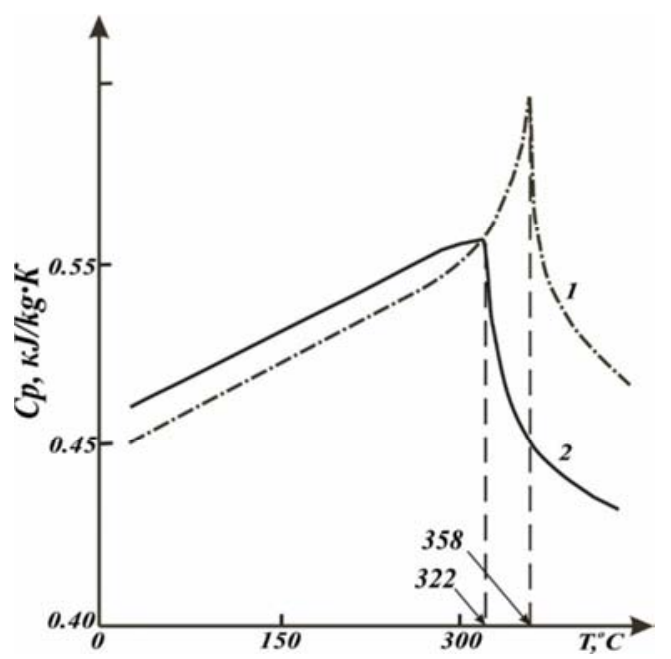

Figure 5. Temperature inter-dependence of the specific heat of nickel: 1reference data, 2-sample of nanocrystalline $\mathrm{Ni}(70 \mathrm{~nm})$.

The presence of vacancy-cluster structure in $\mathrm{Ni}(70 \mathrm{~nm})$ essentially affects to its magnetic characteristics. Thus, the 
authors in the [17] pointed out noticeable decreases of the Curie point in compact samples of nanocrystalline Ni (Figure 5). The heat capacity temperature dependence of the investigated $\mathrm{Ni}(70 \mathrm{~nm})$ sample, as well as the reference dependence, has an anomaly corresponding to the 2nd kind phase transition - ferromagnetic-paramagnetic transition. According to the reference data the temperature of this transition for nickel $-\mathrm{Tc}=358^{\circ} \mathrm{C}$. For the sample Ni $(70 \mathrm{~nm})$ it was significantly lower-Tc $=322^{\circ} \mathrm{C}$. The some smoothing of the $\mathrm{C}_{\mathrm{p}}(\mathrm{T})$ anomaly dependence is observed, apparently, due to the imposition of the recrystallization process on the transition phase accompanied by heating releases.

\section{Creation of a Vacancy Cluster Tubes (VCT) Ordering Substructure at High-Intensity Plastic Deformation (HIPD) of Metals and Their Properties Changes}

Based on the physical principles, stated above, obtained

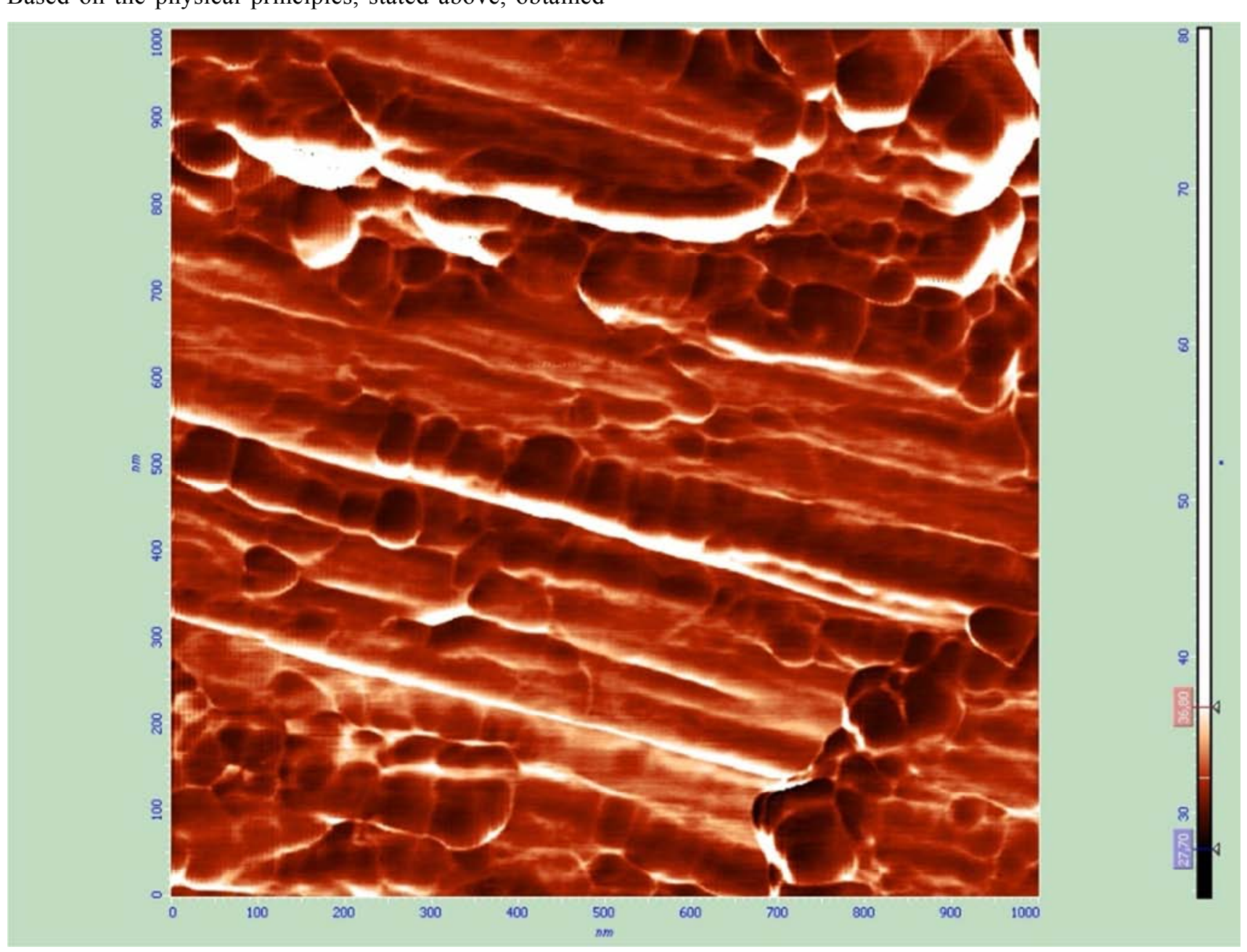

Figure 6. Microstructure of the thin section surface with vacancy cluster tubes of Pb-VCT sample, obtained by atomic force microscopy (AFM) using a Femto Skan and Solver probe microscopes, P. 47. by thermomechanical severe plastic deformation (SPD) structured metals, the authors in [18-20] assigned task (goal) to realize the dimensional effect of dynamic recrystallization [1-4] in the mode of high-intensity plastic deformation (HIPD) in the metal at the moment of its mass crystallization. To solve this problem a centrifugal casting machine of a new design was made. The vertical centrifugal casting machine designed with a rotary speed-2000 r. p. m. The high-intensity plastic deformation (HIPD) mode in the solid hardened phase of the crystallized metal is possible to create by these high rotary speeds. The shifting deformation in the designed machine occurs through the creation of large gradient of the temperature field from the periphery (relatively of the rotor's cold wall) to the molten rotor's central part against the background of high-intensity rotation. The difference between the angular velocities of the already hardened metal part (adjacent to the outer surface of the rotor wall) and the central part where the metal still in the molten state leads to a high-intensity deformation $\left[\varepsilon^{\prime}=\left(10^{2}-10^{4}\right) \mathrm{sec}^{-1}\right]$ of the crystallized metal's solid hardened phase. 
Since the grain sizes of the crystallized phase at the initial moment are about tens of nanometers (the order of the size of the crystallization embryo), it is possible to realize the dimensional effect of "nanocrystalline" hardened metal dynamic recrystallization [1-4] at high shear deformation rates. Experiments in $[18,20]$ were carried out on $\mathrm{Al}$ and $\mathrm{Pb}$.
The mass of the material ranged from 50 to $200 \mathrm{~kg}$.

Crystallization of $\mathrm{Al}$ and $\mathrm{Pb}$ metal melts with a given mass and initial melt temperature carried out under their volumetric supercooling at unsteady conditions (highintensity plastic deformation) with time-specified modes of centrifugal forces and temperatures action.

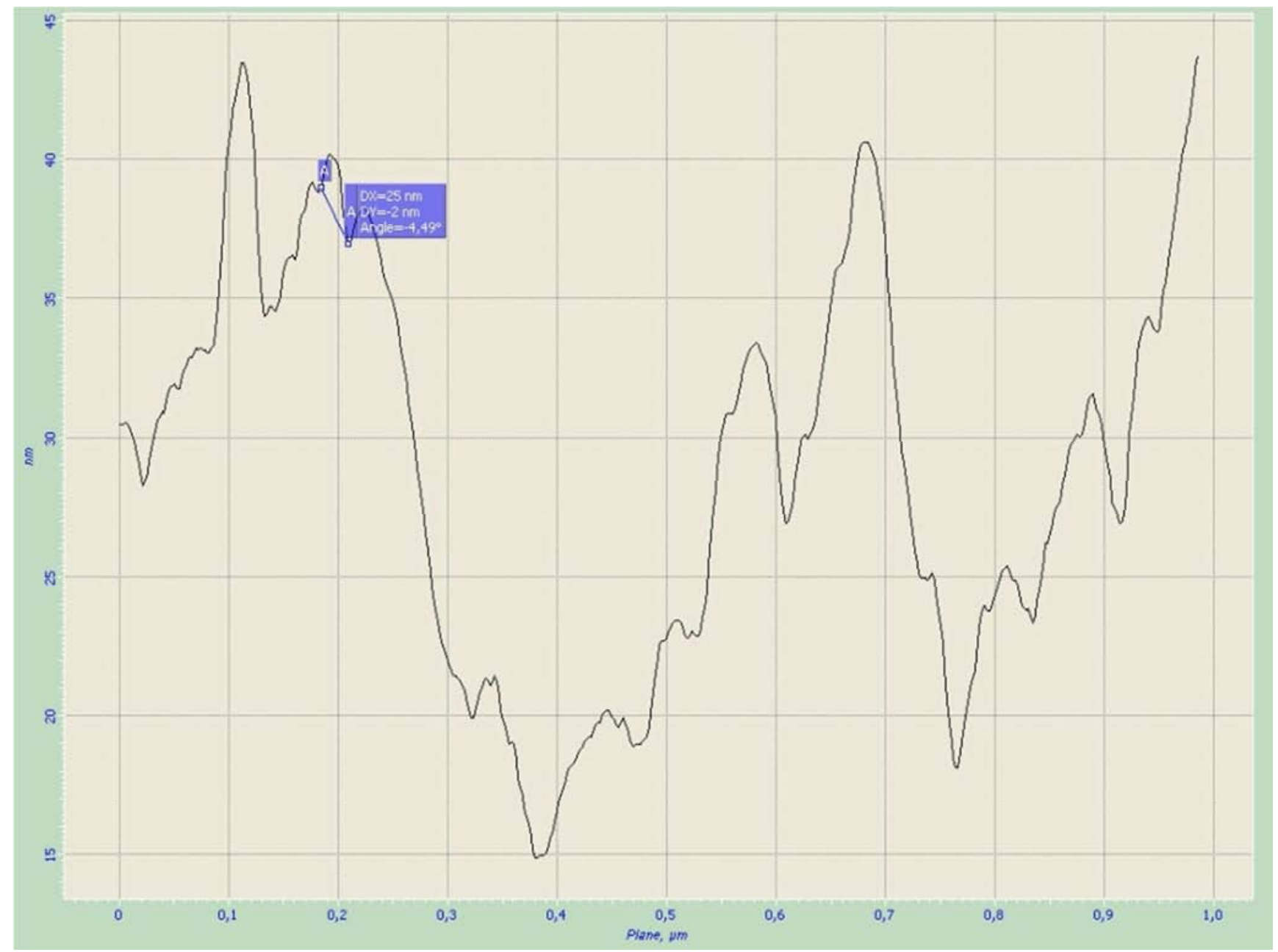

Figure 7. Surface relief of the Pb-VCT sample with vacancy cluster tubes images. The characteristic surface scales are determined using a power spectrum (Fourier analysis), Femtoscan online software applied

In Figures 6-10 the structural studies results of the obtained metals are represented. As evident from the represented data in the amount of the crystallized $\mathrm{Al}$ and $\mathrm{Pb}$ obtained a new type of vacancy structures in the form of cluster tubes (VCT) with the average diameters for Al $39 \mathrm{~nm}$ and for $\mathrm{Pb}-25 \mathrm{~nm}$. The tubes elongated to the rotary center (Figures 6, 8, 9). This form of VCT symmetry indicates that their formation occurs in the crystallized region of the sample.

Estimated concentration of nonequilibrium vacancies gives the values in range $-10^{-3}-10^{-2}$. Such high concentration of nonequilibrium vacancies, as its mentioned above, leads to their condensation into vacancy cluster tubes (VCT) with hexagonal symmetry.

On the other hand, high stationary concentration of nonequilibrium vacancies creates the possibility of solid- phase recrystallization of the metal following the primary crystallization. The process proceed in conditions that is far from the equilibrium in comparison with the usual crystallization of the metal from the melt. These kind of processes can lead to the formation of high-order nonequilibrium structures characterized for nonequilibrium open systems (such as dissipative structures).

Thus the conclusion here is that the high-intensity plastic deformation (HIPD) at the stage of melt crystallization against the background of high stationary concentration of nonequilibrium vacancies occurs the formation of a new type of structure elements - vacancy cluster tubes (VCT). 


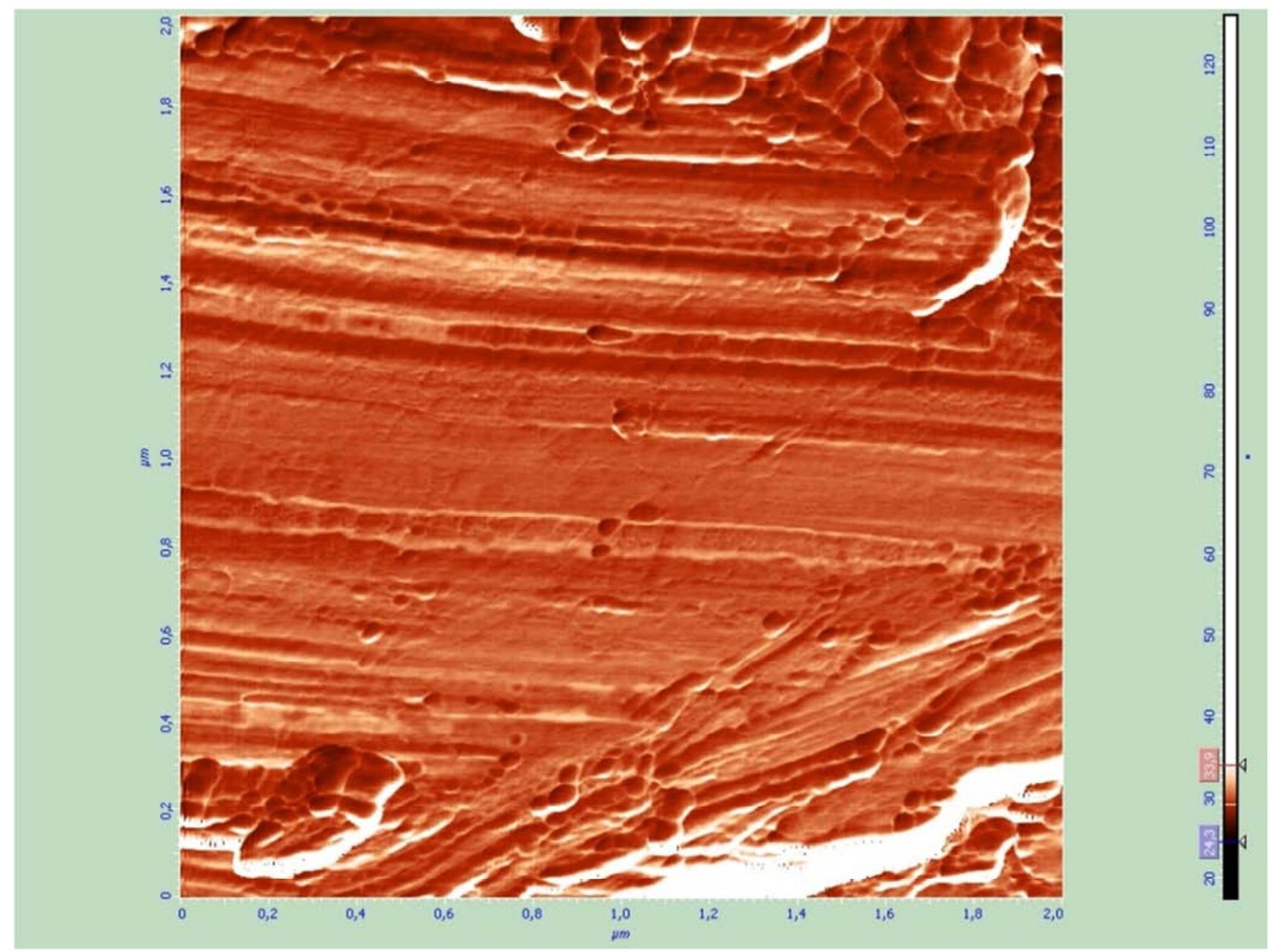

Figure 8. Image of the vacancy cluster tubes surfaces of the Al-VCT sample, obtained using the method of atomic force microscopy (AFM) on the probe microscope Femto Skan and Solver, P47.

Physical processes have the following interpretation: The mechanical energy dissipation in the thin transition "solid liquid" zone with condition of high-intensity shear deformation [18-20] promotes the formation of highly concentrated nonequilibrium vacancies (about $\sim 10^{-2}$ ) in the solidified part of the melt, which creates the possibility for repeated recrystallization of the metal in the solid phase (solid recrystallization phase) in strongly nonequilibrium conditions with simultaneous condensation of nonequilibrium vacancies in the field of centrifugal forces in vacancy clusters of hexagonal tubes forming.

Visual analysis of regulated VCT structures (Figure 6-10) shows two main qualitative features of the new substructure. The first is the thickness of the VCT's walls is about 1-2 nm (!!) that corresponds to the similarity of two-dimensional NMM which properties are well studied in [1-6]. One of the main features of such thin-walled NMS is the absence of dislocations and impossibility of their occurrence as it mentioned above [11-13].

Consequently, the deformation hardening in such structures (VCT) by the dislocation mechanism should be absents and plastic deformation, as noted above, can occur only on the diffusion - viscous mechanism. The second feature is the "Laplacian" tensile stresses in the thin metal walls between the tubes for the small diameters of the VCT (several tens of nanometers), as calculations show, can reach values up to $500 \mathrm{~atm}$ which should lead to increments of the lattice parameter and appearance of extensive elastic stresses.

Such stresses possibly described as a special type of metals hardening with a regular regulated VCT structure. This should primarily essentially affect to the metals mechanical properties.

In Figure 11, 12 the results of mechanical tensile tests of $\mathrm{Pb}$ and $\mathrm{Al}$ [21-23] are represented. As follows from the represented results, there are significant changes in the basic mechanical characteristics of the structured $\mathrm{Pb}$ and $\mathrm{Al}$ metals compared to the initial samples. Thus, for $\mathrm{Pb}$ the elastic limit increases on $60 \%$ and for $\mathrm{Al}$ on $30 \%$, the tensile strength more than $80 \%$ for $\mathrm{Pb}$ and on $40 \%$ for $\mathrm{Al}$. In this case, the relative elongation of the structured $\mathrm{Pb}$ and $\mathrm{Al}$ samples without destruction (plasticity) increases in 2-3 times compared to the original samples. 


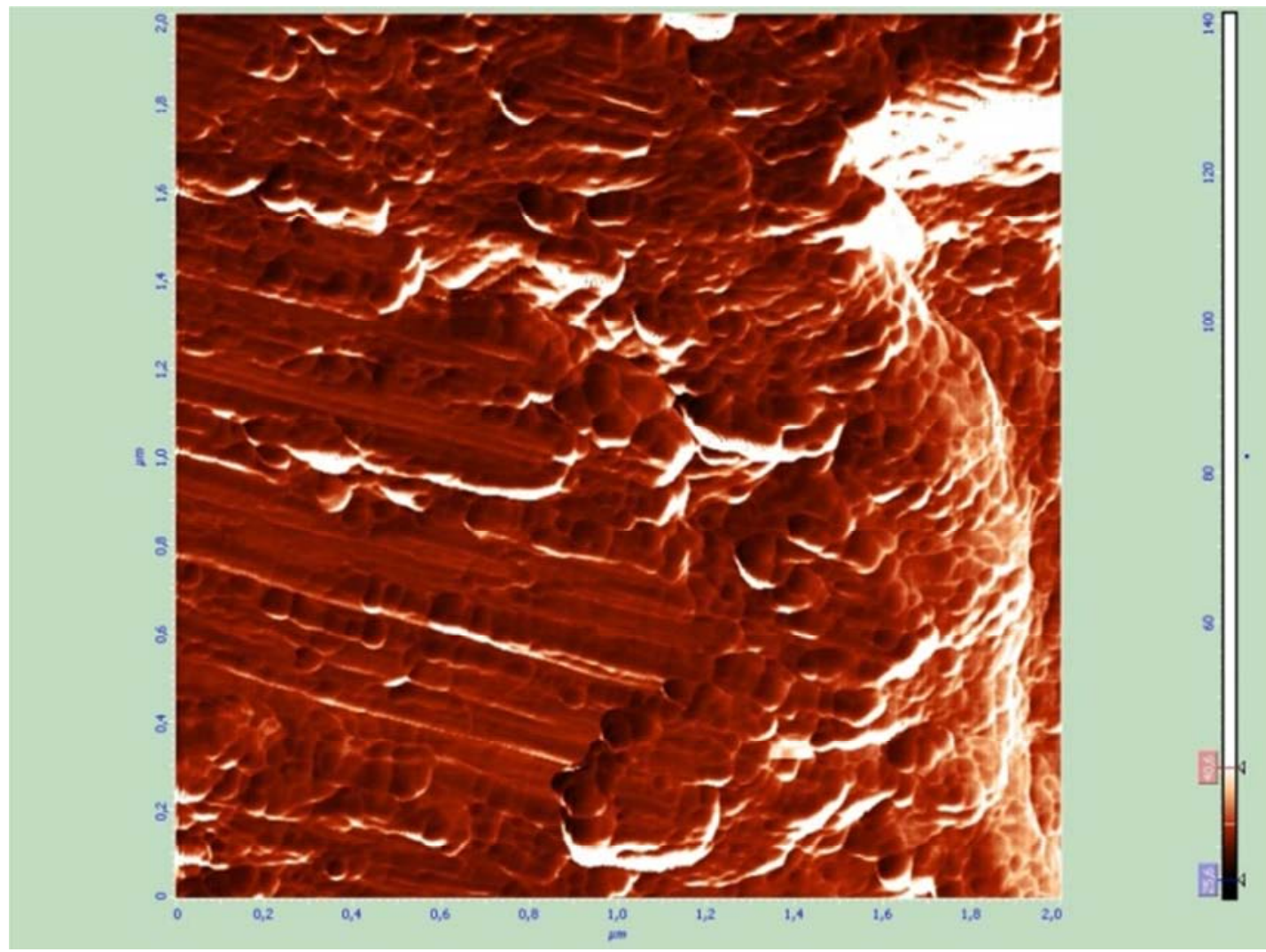

(a)

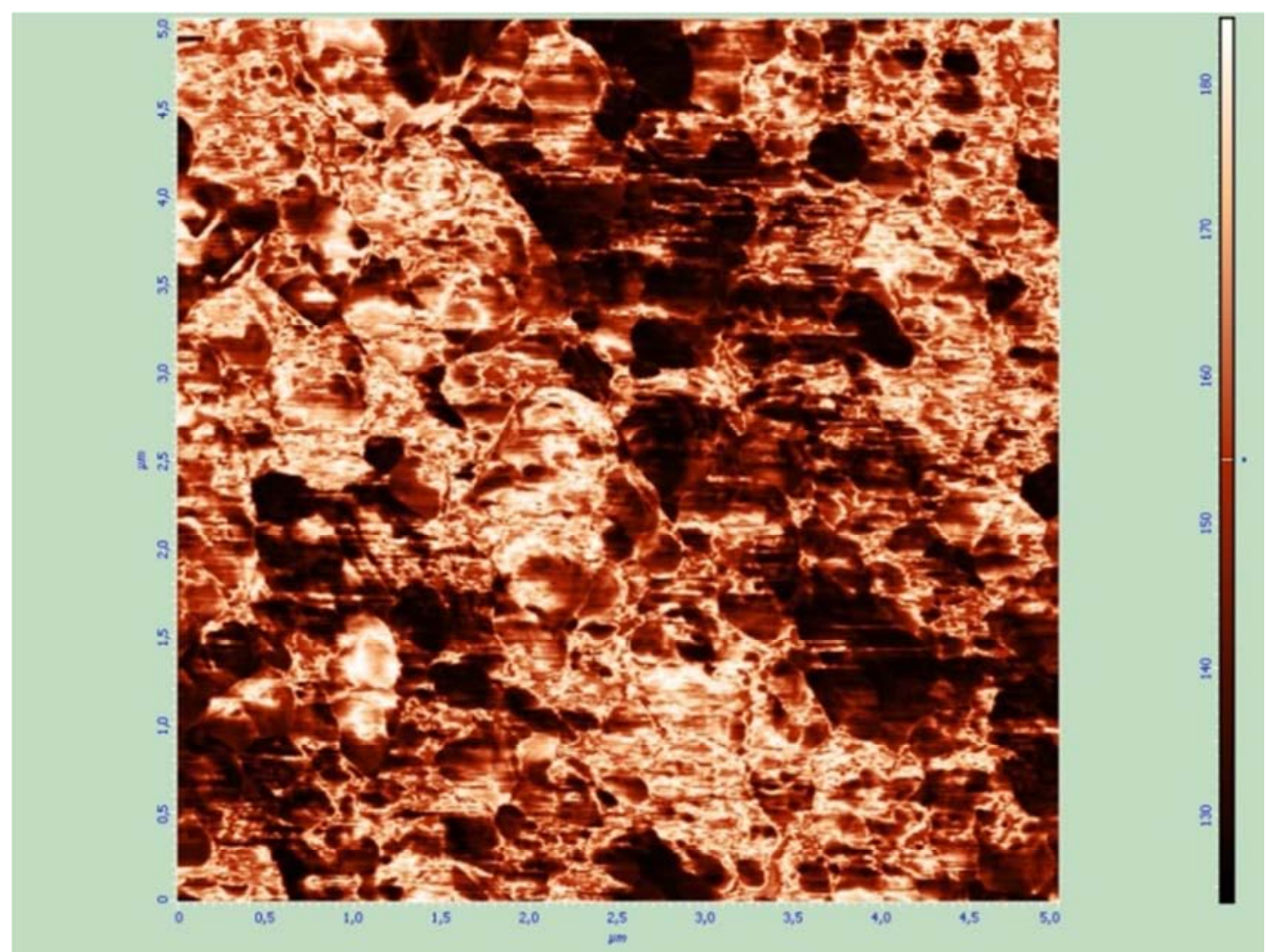

(b)

Figure 9. Microstructure of the vacancy cluster tubes surface of the Al-VCT sample, obtained with atomic force microscopy (AFM) using a Femto Skan and Solver probe microscope, P47; A) - break in the section microstructure; B) - end of the section microstructure. 


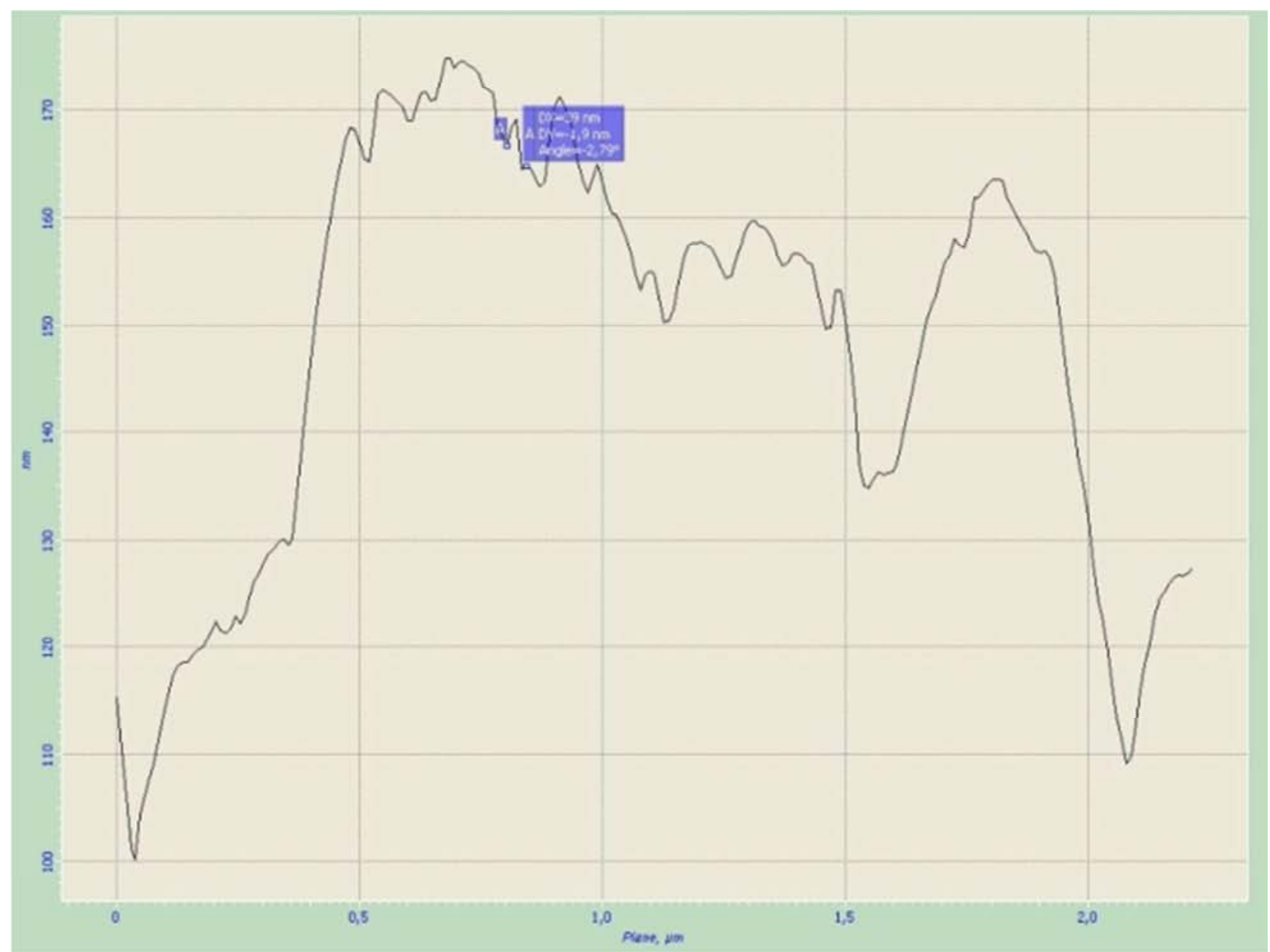

Figure 10. Surface relief of the Al-VCT sample with vacancy cluster tubes. The characteristic surface scales were determined using a power spectrum (Fourier analysis) with the Femtoscan online software applied.

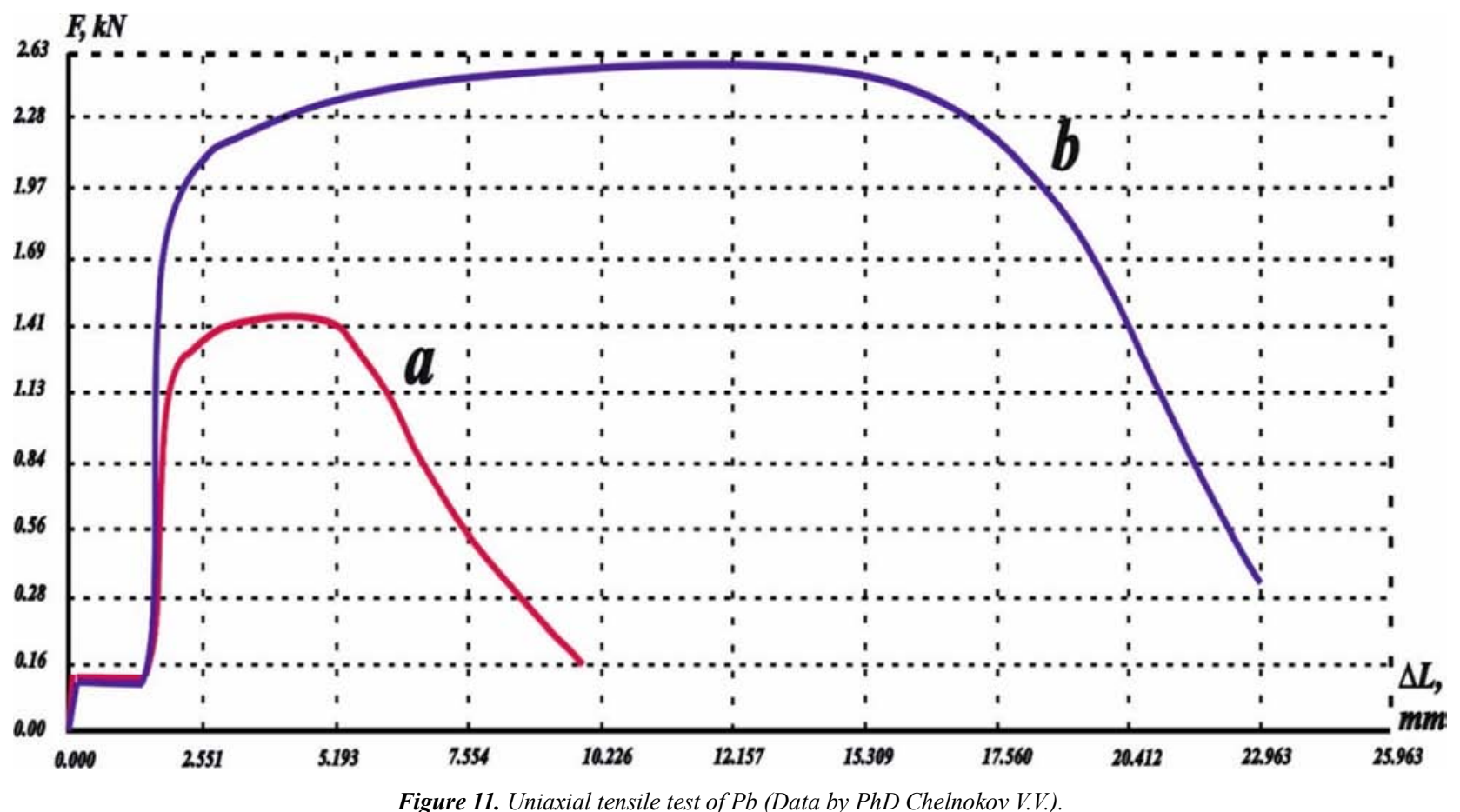




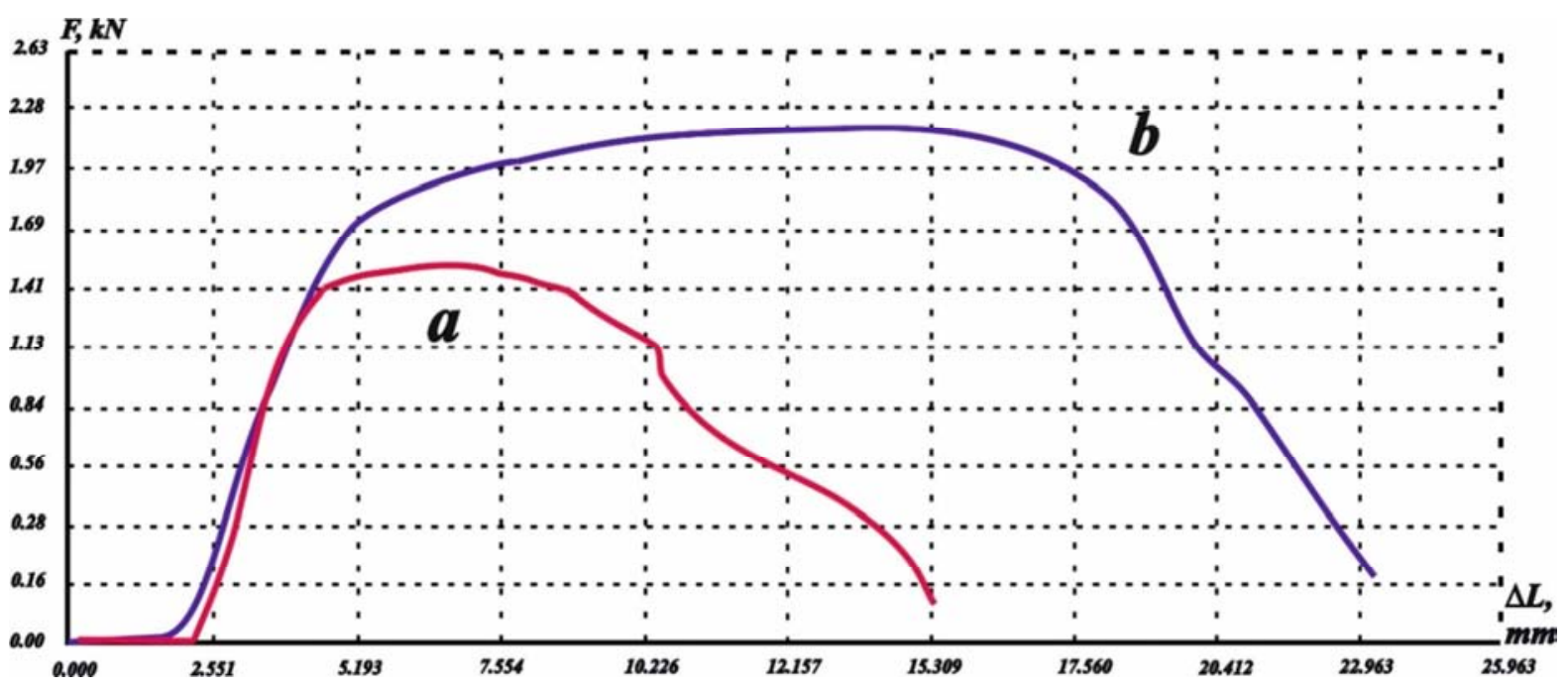

Figure 12. Uniaxial tensile test of Al (Data by PhD Chelnokov V.V.).

In Figure 13 the results of the elastic modulus, the loss modulus and the tangent of a loss angle (internal friction) dependences measuring by the temperature for $\mathrm{Pb}$ samples created with alternating load modes accordingly to dynamicmechanical analysis (DMA) method are represented.

The elasticity modulus on the structured sample is in 4.5 times higher than on the initial one. In the pre-melting temperature range, the modulus of elasticity value on the structured sample remains twice high as on the initial sample at room temperature. This result directly evidenced that the $\mathrm{Pb}$ VCT structure maintaining up to the melting point.

It is obvious that the high-temperature stability of the VCT structures is because they occur at the metal's crystallization temperatures and subsequently remain stable when cooled.

A comparison of the losses module values indicates a fivefold increases of the damping strength in structured $\mathrm{Pb}$. The tangent of the loss angle (interatomic internal friction) in the original $\mathrm{Pb}$ sample is more than two times higher than for structured $\mathrm{Pb}$ in all investigated temperatures interval (Figure 13). This indicates that in the structured $\mathrm{Pb}$ a dissipation under the cyclic loads realized mainly due to elastic relaxation.

Such features of energy dissipation under cyclic loads gives structured metals unique damping properties that can significantly increase the resistance to fatigue failure and successfully counteract to resonance phenomena. In Figure 14 the magnetic permeability temperature dependences for the samples of standard $\mathrm{Pb}$ ("initial") with a large-crystal structure and $\mathrm{Pb}$ ("No.1) after solidification in centrifugal casting with speed 2000 r. p. m.

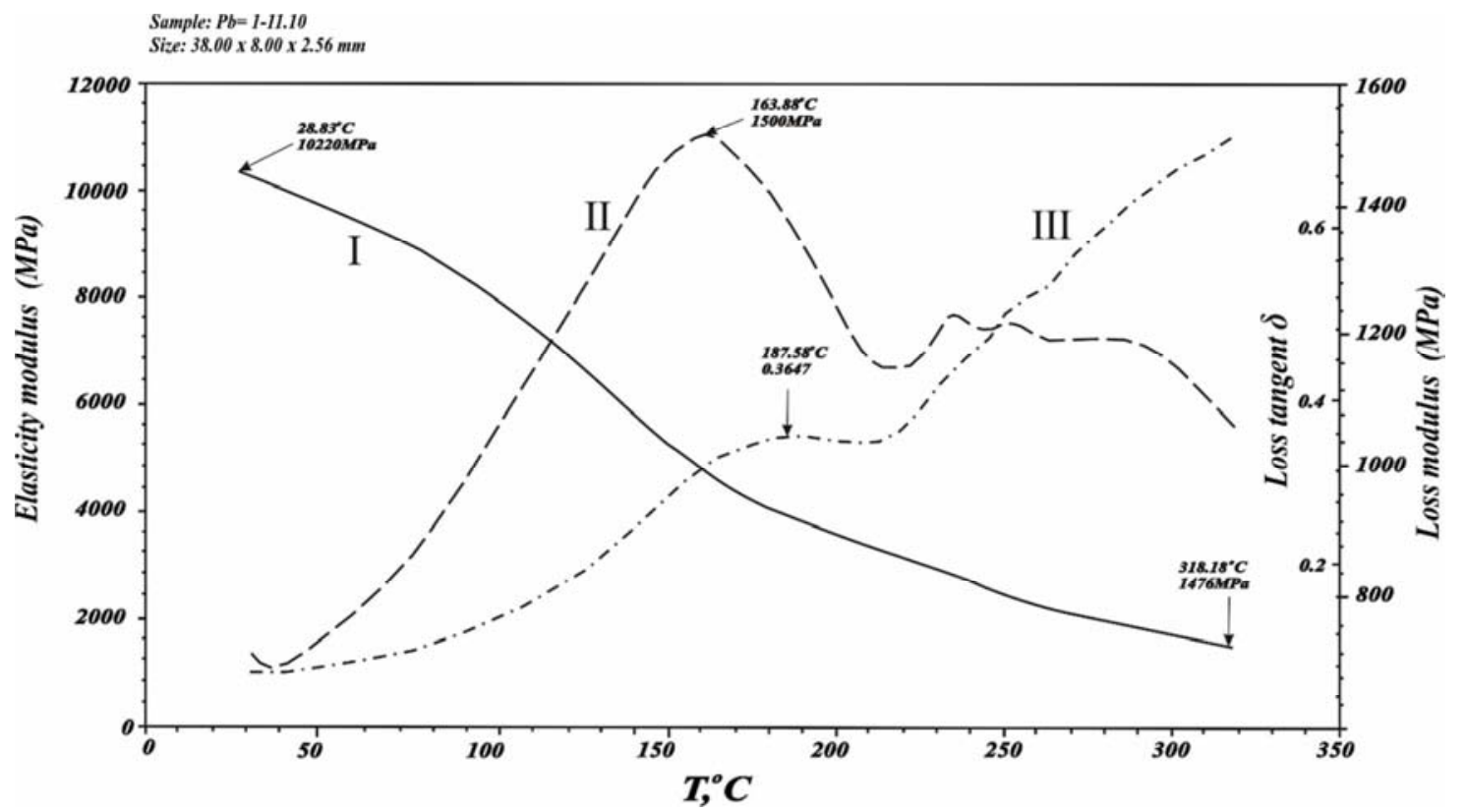




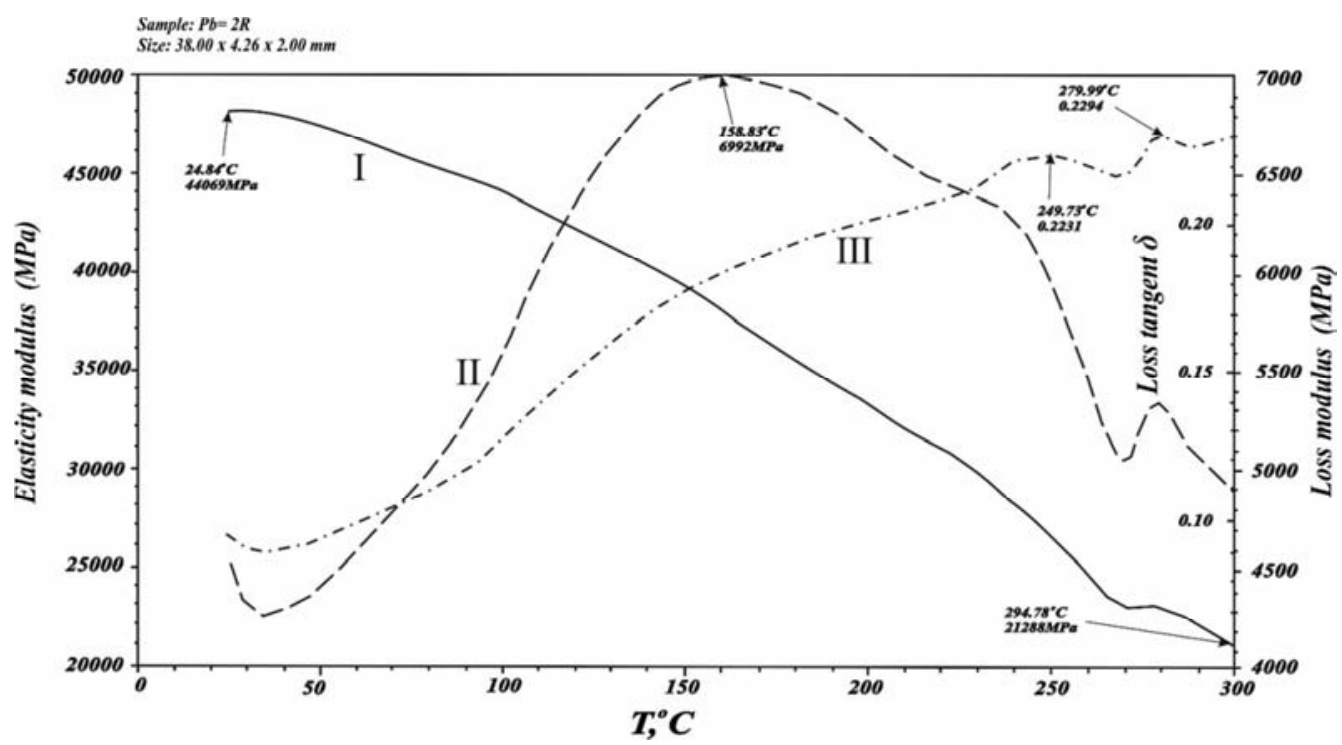

(b)

Figure 13. Thermal $\left(T^{\circ} \mathrm{C}\right)$ dependency graphs of Pb elasticity modulus (I), loss modulus (II) and loss tangent $\delta$ (III). (Data by PhD Chelnokov V.V.). a) initial sample b) structured sample.

From obtained results is follows that the characteristic of the transition to the superconducting state of the $\mathrm{Pb}$ "No.1"sample (upper curve) is different from the $\mathrm{Pb}$ "initial" sample. The graph shows that $\mathrm{Pb}$ is classical example of low-temperature superconductor of type I. The transition to the superconducting state of the $\mathrm{Pb}$ "No.1" sample proceeds accordingly to the superconductors of type II.

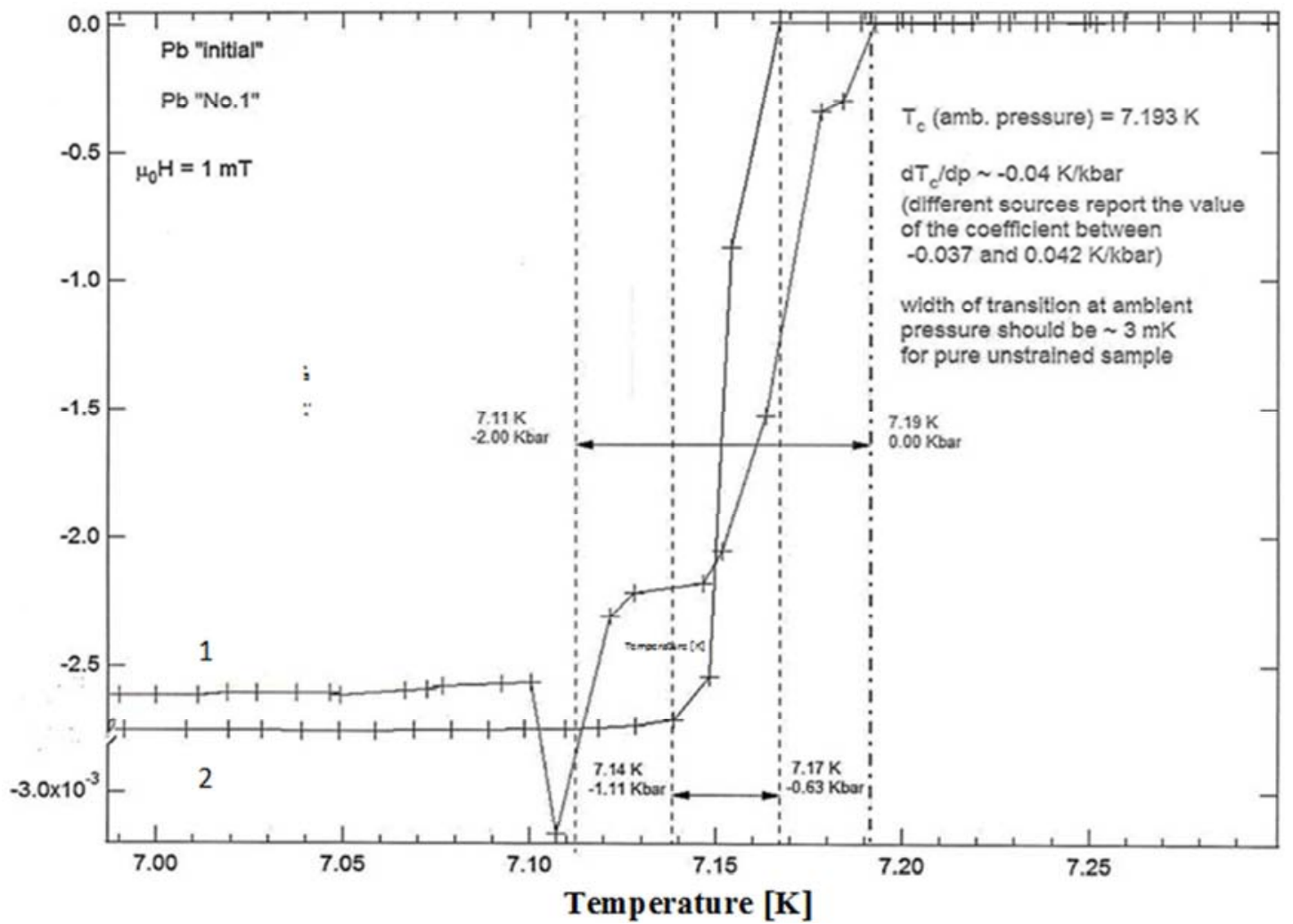

Figure 14. Comparative studies of the nature of the superconducting transition during determining the superconducting transition temperature (Tc) for standard t'nitial"Pb-2 and for $\mathrm{Pb}$ No.1"with VCT-1.

This result is unique not only from viewpoint of the metals new crystallization technology in HIPD, but has a fundamental physical aspect, as typical superconductors of the type II that traditionally includes materials with lattice structures consists of metals and nonmetals atoms.

Thus, using of new HIPD technology makes it possible to 
create from metals some materials with non-metallic or mixed properties.

\section{Conclusion}

The studies and tests of the discussed HIPD technology revealed a wide range of possibilities to create bulkstructured metals with different unique properties and after further processing, make it into finalized products.

Structured metals are perspective for use in the aerospace industry, transport and engineering industries, engine building, microelectronics, power engineering and other high-tech industries.

The structured metals in the form of a self-organizing VCT system created by unique method are rather convincing example of the practical implementation of the models theoretical development for the formation of dissipative structures in open systems [24].

Obtained results suggest the possibility of creating a new scientific direction in material science.

\section{References}

[1] V. I. Novikov, L. I. Trusov, V. G. Gryaznov, etc. "Dimensional effect of recrystallization. Surface" IIPhysics, chemistry, mechanics. 1986. №1, 134-139 pp.

[2] V. I. Novikov, V. N. Lapovok, S. V. Svirida et al. "Formation of nonequilibrium vacancies in ultrafine Nickel powder under plastic flow under pressure" $\mid \backslash$ Physics of metals and metallology. 1984. Vol. 57, Issue 4, 718-721pp.

[3] V. I. Novikov, L. I. Trusov, V. G. Gryaznov, "Solid-phase transformations, initiated by the migrating boundaries" "ISat. The growth of crystals. Red. Givargizov E. I. and Greenberg S. A., Vol. 17 (1988) Science. M. S, 69-86 pp.

[4] L. I. Trusov, V. I. Novikov, Y. A. Lopukhov, V. N. Lapovok, "Recrystallization in ultrafine systems" $\mid \backslash$ Physicochemistry of ultradispersed systems.-M.: Science. 1987. 67-74 pp.

[5] J. E. Geguzin, L. N. Paritskaya, V. V. Bogdanov, V. I. Novikov "The features of recrystallization of ultrafine powder during sintering" \\Physics of metals and metallurgy. 1983. Vol. 55, issue 4, 768-773 pp.

[6] V. N. Lapovok, V. I. Novikov, S. V. Svirida, etc. "Formation of nonequilibrium vacancies during recrystallization of ultrafine powder of Nickel" \|FIZ. 1983. Vol. 25, issue 6, 1846-1848 pp.

[7] S. S. Gorelik, M. S. Blanter, "Education job at recrystallization" $\mid$ Izv. USSR ACADEMY OF SCIENCES. Metals. 1982. №2, 90-93 pp.

[8] G. Gleiter, B. Chalmers, 'Large-Angle grain boundaries" $\mid \backslash \mathrm{M}$.: World, 1975. $374 \mathrm{p}$.

[9] V. M. Ievlev, "Thin films of inorganic materials: growth mechanism and structure: studies", I|Benefit. - Voronezh: Ed. VSU. 2008. 496 p.

[10] W. M. Kosevich, V. M. Ievlev, L. S. Palatnik, A. I. Fedorenko, "Structure of intergranular and interphase boundaries" IIM.: Metallurgy. 1980. $256 \mathrm{p}$.
[11] V. I. Novikov and V. Ya. Ganelin, L. I. Trusov, etc. "The Effect of dilatation in the ultrafine crystal recrystallization in Nickel” |ISolid State Physics. 1986. Vol. 28, №4, 1251-1254 pp.

[12] J. Friedel, "Dislocations" ||M.: World. 1967. 643p.

[13] V. I. Novikov, V. Ya. Ganelin, L. I. Trusov, etc. "Inhibition of recrystallization of ultrafine Ni powder under high hydrostatic pressure" $\mid \backslash$ Physics of metals. 1986. Vol. 8. B, 2p. 111-113 pp.

[14] L. I. Trusov, V. I. Novikov, I. A. Repin, E. E. Kazilin, V. Ya. Ganelin, "Deformation of Ni ultrafine structure" \|Physics of metals. 1988. Vol. 10, №1, 104-107 pp.

[15] L. I. Trusov and T. P. Khvostantseva, V. A. Solov'ev, V. A. Mel'nikova, "Low temperature stress relaxation of nanocrystalline nickel" \\Journal of Materials Science. 1995. V. 30, №11, pp. 2956-2961.

[16] L. I. Trusov, T. P. Khvostantseva, V. A. Solov'ev, V. A. Mel'nikova, "Stress relaxation following heating of nanocrystalline nickel" |INanostructured Materials. 1994. V. 4, №7, 803-813 pp.

[17] R. Z. Valiev, Z. Z. Mulikov, H. Y. Mulikov, V. I. Novikov, L. I. Trusov, "Curie temperature and saturation magnetization of Nickel with subgrain structure" $\mid \backslash$ Technical physics letters. 1989. Volume 15, vol. 1, 78-81 pp.

[18] Y. I. Tarasov, V. V. Kryachko, V. I. Novikov, "Development of new structured materials for aerospace industry" IISat. scientific. article on the materials of the $\mathrm{V}$ International scientific.- prakt. Conf. "Academic Zhukovsky reading". 2223 Nov 2107. Voronezh: VUNTS VVS "VVA". 2018. 255-257 pp.

[19] Y. I. Tarasov, V. V. Kryachko, V. I. Novikov, "Structuring Features at mass crystallization of melts $\mathrm{Al}$ and $\mathrm{Pb}$ under conditions of high-intensity plastic deformation during centrifugation" $\|$ Condensed matter and interphase boundaries. 2018. Vol. 20, №16, 125-134 pp.

[20] Y. I. Tarasov, V. V. Kryachko, V. I. Novikov, "Peculiarities of structuring during the process of mass crystallization of $\mathrm{Al}$ and $\mathrm{Pb}$ melts under conditions of high-intensity plastic deformation during centrifugation" " Book of abstract of the XIV International Conference on Nanostructured Materials (NANO 2018) 24-29 June, 2018. "City University of Hong Kong", 57 p.

[21] V. I. Novikov, "Peculiarities of structuring during the process of mass crystallization of $\mathrm{Al}, \mathrm{Pb}, \mathrm{Zn}$ melts under conditions of high-intensity plastic deformation (HIPD) during centrifugation" ||2018 Russia Advanced Technology Transfer Matchmaking Conference and ACCICB International ST Project Meeting. Sep. 25, 2018. Deijing. 12-15 p.

[22] V. I. Novikov, B. V. Spitsyn, E. M. Soloviev and others, "The physical model of the formation of vacancy cluster of tubes and change the properties of metals at dynamic centrifugal casting" \International scientific journal "Alternative energy and ecology". 2016. № 15-18 (203-208), 96-103 pp.

[23] Y. I. Tarasov, V. V. Kryachko, V. I. Novikov, "The Vacancy Cluster Tubes Formation and Metal Properties Changes After Dynamic Centrifugal Casting" \\American Journal of Modern Physics. 2018. Vol. 7, № 6, 194-202 pp.

[24] Haken G. Synergetics, "Hierarchies of instabilities in selforganizing systems and devices". IIM.: World. 1985. $419 \mathrm{p}$. 